Pacific Journal of Mathematics

GENERALIZED SOLUTIONS FOR THE MEAN CURVATURE
EQUATION 


\section{GENERALIZED SOLUTIONS FOR THE MEAN CURVATURE EQUATION}

\section{EnRICO GIUsti*}

The purpose of this paper is to discuss general boundary valve problems for the mean curvature equation

$$
\operatorname{div} T u=H(x, u) ; \quad T u=D u / \sqrt{1+|D u|^{2}}
$$

in a bounded domain $\Omega \subset \boldsymbol{R}^{n}$. More precisely, we shall consider the problem of minimizing the functional

$$
\mathscr{F}(u)=\int_{\Omega} \sqrt{1+|D u|^{2}}+\int_{\Omega} \lambda(x, u) d x+\int_{\partial \Omega} \kappa(x, u) d H_{n-1}
$$

where

$$
\lambda(x, u)=\int_{0}^{u} H(x, t) d t .
$$

It is easily seen that (0.1) is the Euler equation of the functional $\mathscr{F}$. The third integral in (0.2) describes the boundary conditions: if $u \in C^{1}(\bar{\Omega})$ and $\kappa$ is of class $C^{1}$ we have

$$
T u \cdot \nu=\gamma(x, u)
$$

on $\quad \partial \Omega$

where $\nu$ denotes the interior normal to $\partial \Omega$, and

$$
\kappa(x, u)=\int_{0}^{u} \gamma(x, t) d t .
$$

When $\kappa$ is not differentiable, as it is the case for the Dirichlet problem, condition 0.4 does not hold any longer, and we have instead the weaker condition

$$
\gamma^{-}(x, u) \leqq T u \cdot \nu \leqq \gamma^{+}(x, u)
$$

on $\partial \Omega$, where

$$
\gamma^{ \pm}(x, u)=\lim _{t \rightarrow u \pm} \gamma(x, t) .
$$

For example, for the Dirichlet problem with boundary datum $f(x)$ we have

$$
\kappa(x, u)=|u-f(x)|-|f(x)|
$$

and

$$
\gamma(x, t)=1-2 \varphi_{F}(x, t)
$$

where $\varphi_{F}$ is the characteristic function of the subgraph $F$ of $f$ : 


$$
\varphi_{F}(x, t)=\left\{\begin{array}{lll}
1 & \text { if } & t<f(x) \\
0 & \text { if } & t \geqq f(x) .
\end{array}\right.
$$

In this case we get the boundary conditions

$$
T u \cdot \nu=\left[\begin{array}{c}
1 \text { if } u(x)>f(x) \\
-1 \text { if } u(x)<f(x) \\
\text { arbitrary if } u(x)=f(x) .
\end{array}\right.
$$

Two standard methods have been developed for the study of boundary value problems. The first one consists in looking for a classical solution, i.e., a smooth function $u(x)$ satisfying equation (0.1) and the boundary conditions (0.4), or more generally (0.6). Alternatively, one may try to minimize directly the functional $(0.2)$ in $B V(\Omega$, the space of functions with bounded variation in $\Omega$.

Both such approaches suffer serious limitations; in particular the variational method is not adequate when dealing with problems whose solutions may have infinite area, as it is the case for the Dirichlet problem with infinite data or in unbounded domains.

Recently, M. Miranda [10] has introduced the notion of generalized solutions for the minimal surface equation, and has used it successfully in the Dirichlet problem in infinite domains [10], and in the problem of removable singularities [11], two questions in which the area of the solution is not finite, at least in principle. The same notion of generalized solution has been used by U. Massari [8] in his paper on Dirichlet's problem with infinite data, and by the author [7] in the problem of maximal domains for the mean curvature equation.

The idea of generalized solutions originates from the observation that a function $u \in B V(\Omega)$ is a variational solution of $\mathscr{F}$ if and only if its subgraph

$$
U=\{(x, t) \in \Omega \times \boldsymbol{R}: t<u(x)\}
$$

minimizes the functional

$$
F(U)=\int_{\Omega \times \boldsymbol{R}}\left|D \mathcal{P}_{l}\right|+\int_{\varrho \times \boldsymbol{R}} H \mathcal{P}_{\ell} d x d t+\int_{\partial \Omega \times \boldsymbol{R}} \gamma \mathcal{P}_{l} d H_{n} .
$$

The subgraph of $u$ has the property that the intersection of any vertical straight line with $U$, if it is not empty, is either the whole line or a lower half-line. Conversely, every set $U$ with the above property is the subgraph of a function $u(x)$, taking possibly the values $+\infty$ or $-\infty$. Such a function is called a generalized solution if $U$ is a local minimum of $F$.

The interest in the above definition comes mainly from the fact 
that under extremely mild hypotheses on $H$ and $\gamma$, the set of generalized solutions is compact. More precisely, from every sequence $u_{j}$ of generalized solutions it is possible to extract a convergent subsequence, in the sense that the subgraphs $U_{j}$ converge locally in $\Omega \times \boldsymbol{R}$ to a subgraph $U$ (Lemma 1.2). Of course, the same is not true for variational solutions, for which one needs at least a uniform estimate in $L_{10 \mathrm{c}}^{1}(\Omega)$.

This paper deals with generalized solutions for the functional (0.2). In the first place we show the existence of such solutions, under very general assumptions for $H$ and $\gamma$. Of course, generalized solutions may take the values $\pm \infty$; in other words the sets

$$
P=\{x \in \Omega: u(x)=+\infty\}
$$

and

$$
N=\{x \in \Omega: u(x)=-\infty\}
$$

may be nonempty. In $\S 2$ we study the properties of these singular sets, showing that they must minimize two functionals related to $F$.

In $\S 3$ we discuss more closely the relations between generalized and variational solutions and we prove that under suitable assumptions the sets $P$ and $N$ are empty and therefore the generalized solutions are variational. In the same section we show how a number of problems treated by various authors may find their natural place in this general setting.

As an application, we discuss in $\S 4$ Lagrange multipliers. This problem has been studied by C. Gerhardt [4] in the case of capillarity, and by G. Williams for Dirichlet's boundary conditions [12], in the framework of variational solutions. The existence of variational solutions being not guarateed in principle by the hypotheses, both authors introduce a perturbed functional (for which existence is granted) and then let the perturbation vanish. The use of the notion of generalized solutions, avoiding this complication and dealing directly with the original functional, permits a considerable simplification of the proof, and a generalization of the results.

1. Existence of generalized solutions. Throughout this paper we shall be concerned with the functional

$$
\mathscr{F}(u)=\int_{\Omega} \sqrt{1+|D u|^{2}}+\int_{\Omega} \lambda(x, u) d x+\int_{\partial \Omega} \kappa(x, u) d H_{n-1}
$$

where $\Omega$ is a bounded domain in $\boldsymbol{R}^{n}$ with smooth boundary $\partial \Omega$, and $\lambda, \kappa$ are convex functions of $u$. We may suppose that $\lambda(x, 0)=$ $\kappa(x, 0)=0$, and therefore 


$$
\lambda(x, u)=\int_{0}^{u} H(x, t) d t ; \quad \kappa(x, u)=\int_{0}^{u} \gamma(x, t) d t
$$

for some functions $H, \gamma$, nondecreasing in $t$ for almost every $x$.

Related to (1.1) we define a second functional, operating on subsets of the cylinder $Q=\Omega \times \boldsymbol{R}$.

For $T>0$ let us set

$$
\begin{aligned}
Q_{T} & =\Omega \times[-T, T] \\
o Q_{T} & =\partial \Omega \times[-T, T],
\end{aligned}
$$

and for $U \subset Q$ :

$$
\text { (1.2) } F_{T}(U)=\int_{Q_{T}}\left|D \varphi_{U}\right|+\int_{Q_{T}} H(x, t) \varphi_{U} d x d t+\int_{\partial Q_{T}} \gamma(x, t) \rho_{U} d H_{n} .
$$

DeFinition 1.1. A set $A \subset Q$ is a supersolution in $Q_{T}$ for the functional $F$ if for every set $S \subset Q_{T}$ we have

$$
F_{T}(A) \leqq F_{T}(A \cup S) .
$$

The set $A$ is a subsolution in $Q_{T}$ if for every set $S \subset Q_{T}$ :

$$
F_{T}(A) \leqq F_{T}(A-S) .
$$

Finally, $A$ is a solution in $Q_{T}$ if it is both a super-and a subsolution.

Definition 1.2. A set $A \subset Q$ is a local solution for $F$ [supersolution, subsolution] in $Q$ if it is a solution [supersolution, subsolution] in $Q_{T}$ for every $T>0$.

The connection between the functionals (1.1) and (1.2) is apparent from the following theorem.

Theorem 1.1. (M. Miranda [9]) A function $u \in B V(\Omega)$ is a variational solution [supersolution, subsolution] for if and only if its subgraph

$$
U=\{(x, t) \in Q: t<u(x)\}
$$

is a local solution [supersolution, subsolution] for $F$ in $Q$.

The above result is the starting point for the definition of generalized solutions for the functional (1.1). Suppose we have a local solution $U$ of the functional $F$, and that almost every vertical line crosses the boundary of $U$ at most once. Then $U$ will be the subgraph of some function $u(x)$ in $\Omega$, taking possibly the values $+\infty$ or $-\infty$. We call such function $u$ a generalized solution for the 
functional $\mathscr{F}$.

Definition 1.3. (M. Miranda [10]) A function $u: \Omega \rightarrow[-\infty,+\infty]$ is a generalized solution [supersolution, subsolution] for the functional $\mathscr{F}$ if its subgraph $U$ is a local solution [supersolution, subsolution] of the related functional $F$.

We shall devote the rest of this section to the proof of the existence of a generalized solution of $\mathscr{F}$.

Lemma 1.1. Let $\partial \Omega$ be a $C^{2}$ manifold. For each $\varepsilon>0$ there exists a constant $c_{1}(\varepsilon)$ such that for every $w \in B V(\Omega)$ we have

$$
\int_{\partial \Omega}|w| d H_{n-1} \leqq \int_{\Sigma_{\varepsilon}}|D w|+c_{1} \int_{\Sigma_{\varepsilon}}|w| d x
$$

where

$$
\Sigma_{\varepsilon}=\{x \in \Omega: \operatorname{dist}(x, \partial \Omega) \leqq \varepsilon\}
$$

Proof. Suppose first that $w \geqq 0$, and let $\eta$ be a $C^{\infty}$-function, $0 \leqq \eta \leqq 1, \eta=1$ on $\partial \Omega$ and $\eta=0$ in $\Omega-\Sigma_{\varepsilon}$. Since $\partial \Omega$ is of class $C^{2}$ the distance function $d(x)=\operatorname{dist}(x, \partial \Omega)$ is of class $C^{2}$ in a neighborhood of $\partial \Omega$. We may suppose of course that $\varepsilon$ is so small that $d \in C^{2}\left(\Sigma_{\varepsilon}\right)$. We have

$$
\int_{\Omega} w \operatorname{div}(\eta D d) d x=-\int_{\Omega} \eta D d D w+\int_{\partial 2} w \eta \nu \cdot D d d H_{n-1}
$$

and since $\nu \cdot D d=-1$ and $\eta=1$ on $\partial \Omega$ :

$$
\int_{\partial \Omega} w d H_{n-1}=-\int_{\Omega} \eta D d D w-\int_{\Omega} w \operatorname{div}(\eta D d) d x \leqq \int_{\Sigma_{\varepsilon}}|D w|+c_{1}(\varepsilon) \int_{\Sigma_{\varepsilon}} w d x
$$

where $c_{1}(\varepsilon)=\sup _{\Omega}|\operatorname{div}(\eta D d)|$ depends only on $\varepsilon$ and $\Omega$. This proves (1.3) when $w \geqq 0$. The general case follows from the inequality

$$
\int_{\Omega}|D| w|| \leqq \int_{\Omega}|D w|
$$

We may now prove

Proposition 1.1. Let the function $\kappa(x, u)$ satisfy

$$
|\kappa(x, u)-\kappa(x, v)| \leqq|u-v|
$$

for $H_{n-1}$ almost every $x \in \partial \Omega$. Then the functional

$$
\mathscr{G}(u)=\int_{\Omega} \sqrt{1+|D u|^{2}}+\int_{\partial \Omega} \kappa(x, u) d H_{n-1}
$$


is lower semi-continuous with respect to $L^{1}$ convergence.

Proof. Let $u_{j} \rightarrow u$ in $L^{1}(\Omega)$. We have from (1.4):

$$
\mathscr{C}(u)-\mathscr{C}\left(u_{j}\right) \leqq \int_{\Omega} \sqrt{1+|D u|^{2}}-\int_{\Omega} \sqrt{1+\left|D u_{j}\right|^{2}}+\int_{\partial \Omega}\left|u-u_{j}\right| d H_{n-1}
$$

and from Lemma 1.1 with $w=u-u_{j}$ :

$$
\begin{aligned}
\varepsilon(u)-\mathscr{C}\left(u_{j}\right) \leqq & \int_{\Omega} \sqrt{1+|D u|^{2}}-\int_{\Omega} \sqrt{1+\left|D u_{j}\right|^{2}}+\int_{\Sigma_{\varepsilon}}|D u| \\
+ & \int_{\Sigma_{\varepsilon}}\left|D u_{j}\right|+c_{1} \int_{\Sigma_{\varepsilon}}\left|u-u_{j}\right| d x \leqq \int_{\Sigma_{\varepsilon}} \sqrt{1+|D u|^{2}} \\
& \quad-\int_{\Omega_{\varepsilon}} \sqrt{1+\left|D u_{j}\right|^{2}}+2 \int_{\Sigma_{\varepsilon}} \sqrt{1+|D u|^{2}} \\
+ & c_{1} \int_{\Sigma_{s}}\left|u-u_{j}\right| d x
\end{aligned}
$$

where

$$
\Omega_{\varepsilon}=\Omega-\Sigma_{\varepsilon}=\{x \in \Omega: \operatorname{dist}(x, \partial \Omega)>\varepsilon\} .
$$

Let now $j \rightarrow \infty$; taking into account the lower semi-continuity of the area with respect to $L^{1}$ convergence we get:

$$
\mathscr{C}(u)-\liminf _{j \rightarrow \infty} \mathscr{C}\left(u_{j}\right) \leqq 2 \int_{\Sigma_{c}} \sqrt{1+|D u|^{2}}
$$

and the result follows letting $\varepsilon \rightarrow 0$.

REMARK 1.1. An assumption equivalent to (1.4) is obviously

$$
|\gamma(x, t)| \leqq 1 \quad H_{n} \text { - a.e. on } \partial Q \text {. }
$$

We have proved the lower semi-continuity of part of the functional $\mathscr{G}(u)$. For what concerns the curvature term we refer to [5] where we have proved its lower semi-continuity with respect to strong convergence in $L^{1}$ and weak convergence in $L^{n / n-1}$, under the hypothesis that $H(x, t)$ is increasing in $t$, and belongs to $L^{n}(\Omega)$ for every $t \in \boldsymbol{R}$.

We have in conclusion the following theorem:

THEOREM 1.2. Let $\Omega$ be a bounded domain with $C^{2}$ boundary $\partial \Omega$, and let $H(x, t)$ and $\gamma(x, t)$ be two functions defined in $Q$ and $\partial Q$ respectively, and satisfying the following assumptions:

$\left(\mathrm{H}_{1}\right) \quad H(x, \cdot)$ is nondecreasing for almost every $x \in \Omega$.

$\left(\mathrm{H}_{2}\right) \quad H(\cdot, t)$ belongs to $L^{n}(\Omega)$ for every $t$.

$\left(\gamma_{1}\right) \quad \gamma(x, \cdot)$ is nondecreasing for $H_{n-1}$-almost every $x \in \Omega$. 


$$
|\gamma(x, t)| \leqq 1 \quad H_{n}-\text { a.e. in } \partial Q
$$

Then for every sequence $u_{j}$, bounded in $L^{n / n-1}$ and convergent in $L^{1}$ to a function $u$ we have:

$$
\mathscr{F}(u) \leqq \liminf _{j \rightarrow \infty} \mathscr{F}\left(u_{j}\right) \text {. }
$$

In order to get a generalized solution for $\mathscr{F}(u)$ we begin by minimizing this functional in the class

$$
V_{j}=\{v \in B V(\Omega):|v| \leqq j\} .
$$

It is easily seen that $\mathscr{F}$ is bounded from below in $V_{j}$, and that every minimizing sequence is bounded in $B V(\Omega)$. From well-known compacteness theorems we may extract a subsequence converging in $L^{1}(\Omega)$; on the other hand it is obvious that sequence will be bounded in $L^{n / n-1}$, so that we may apply Theorem 1.2 to conclude the existence of a minimum for $\mathscr{F}$ in $V_{j}$.

Let us denote by $u_{j}$ a minimizing function. The subgraph $U_{j}$ is a solution for $F$ in $Q_{j}$. We shall now let $j \rightarrow \infty$ to show the existence of a local solution to $F$ in $Q$, and whence of a generalized solution for $\mathscr{F}$. For that we need the following lemma:

Lemma 1.2. Let $A$ be a subsolution for $F$ in $Q_{T}$. Then

$$
\int_{Q_{T}}\left|D \varphi_{A}\right| \leqq c_{2}(T)
$$

Proof. We have

$$
\begin{gathered}
F_{T}(A)=\int_{Q_{T}}\left|D \varphi_{A}\right|+\int_{Q_{T}} H \varphi_{A} d x d t+\int_{\partial Q_{T}} \gamma \varphi_{A} d H_{n} \leqq F_{T}\left(A-Q_{T}\right) \\
=\int_{Q_{T}}\left|D \varphi_{A-Q_{T}}\right| \leqq 2|\Omega|
\end{gathered}
$$

and therefore

$$
\int_{Q_{T}}\left|D \varphi_{A}\right| \leqq 2|\Omega|+\int_{Q_{T}}|H| d x d t+H_{n}\left(\delta Q_{T}\right)=c_{2}(T) .
$$

In a similar way, comparing with $A \cup Q_{T}$, we prove (1.6) for supersolutions. In particular, (1.6) holds for solutions in $Q_{T}$.

The inequality (1.6) is the only estimate we need in order to pass to the limit as $j \rightarrow \infty$. For, let $T>0$ and let $j>T$. Since $U_{j}$ is a solution in $Q_{T}$, we have

$$
\int_{Q_{T}}\left|D \varphi_{U_{j}}\right| \leqq c_{2}(T)
$$


and therefore it is possible to extract a subsequence, which we shall denote again by $U_{j}$, converging to some set $U$ in every $Q_{T}$. It is clear that $U$, being limit of subgraphs, is itself a subgraph of some function $u(x)$, assuming possibly the values $\pm \infty$. It follows from the next proposition that $U$ is a local minimum for $F$ and hence that $u$ is a generalized solution for $\mathscr{F}$.

Proposition 1.2. Let $H_{j}$ and $\gamma_{j}$ be two nondecreasing sequences converging to $H$ and $\gamma$ respectively, and let $U_{j}$ minimize the functional

$$
F_{T}^{j}(A)=\int_{Q_{T}}\left|D \varphi_{A}\right|+\int_{Q_{T}} H_{j} \varphi_{A} d x d t+\int_{i Q_{T}} \gamma_{j} \varphi_{A} d H_{n} .
$$

Suppose that $U_{j} \rightarrow U$ in $Q_{T}$ and that

$$
\int_{\check{\partial} Q_{T}}\left|\varphi_{U_{j}}-\varphi_{U}\right| d H_{n} \longrightarrow 0, \quad \tilde{\partial} Q_{T}=\partial Q_{T}-\delta Q_{T} .
$$

Then $U$ minimizes $F_{T}$ in $Q_{T}$.

Proof. Let $V \subset Q, V=U$ outside $Q_{T}$, and let

$$
V_{j}= \begin{cases}V & \text { in } Q_{T} \\ U_{j} & \text { outside } Q_{T} .\end{cases}
$$

We have $F_{T}^{j}\left(U_{j}\right) \leqq F_{T}^{j}\left(V_{j}\right)$ and therefore

$$
\begin{aligned}
\int_{Q_{T}}\left|D \varphi_{U_{j}}\right| & +\int_{Q_{T}} H_{j} \varphi_{U_{j}} d x d t+\int_{\hat{i}_{T}} \gamma_{j} \varphi_{U j} d H_{n} \\
& \leqq \int_{Q_{T}}\left|D \varphi_{r^{\prime} j}\right|+\int_{Q_{T}} H_{j} \varphi_{V} d x d t+\int_{i Q_{T}} \gamma_{j} \varphi_{V} d H_{n} .
\end{aligned}
$$

On the other hand

$$
\int_{Q_{T}}\left|D \varphi_{V_{j} j}\right| \leqq \int_{Q_{T}}\left|D \varphi_{V}\right|+\int_{\tilde{o}_{Q_{T}}}\left|\varphi_{Q}-\varphi_{V^{\prime} j}\right| d H_{n}
$$

and in conclusion, if $j>s$ :

$$
\begin{gathered}
\int_{Q_{T}}\left|D \varphi_{U_{j}}\right|+\int_{Q_{T}} H_{s} \varphi_{U_{j}} d x d t+\int_{\partial Q_{T}} \gamma \varphi_{U^{j} j} d H_{n}+\int_{\partial Q_{T}}\left(\gamma_{j}-\gamma\right) \varphi_{U j} d H_{n} \\
\leqq F_{T}^{j}(V)+\int_{\tilde{\partial} Q_{T}}\left|\varphi_{U}-\varphi_{l^{\prime} j}\right| d H_{n} .
\end{gathered}
$$

Passing to the limit as $j \rightarrow \infty$ :

$$
\int_{Q_{T}}\left|D \varphi_{U}\right|+\int_{Q_{T}} H_{s} \rho_{U} d x d t+\int_{\partial Q_{T}} \gamma \varphi_{L} d H_{n} \leqq F_{T}(V)
$$


and the conclusion follows letting $s \rightarrow \infty$.

We may now apply the preceding proposition with $H_{j}=H$ and $\gamma_{j}=\gamma$; the condition (1.7) being satisfied for almost every $T$. In conclusion, we have proved the following existence theorem:

THEOREM 1.3. Let $\Omega, \gamma$ and $H$ satisfy the hypotheses of Theorem 1.2. Then there exists a generalized solution for the functional $\mathscr{F}(u)$.

REMARK 1.2. Though proved for free solutions, the above theorem remains valid if some additional conditions are imposed on $u$. In particular, the same proof works for the obstacle problem, i.e., when $u$ is restricted by the conditions

$$
\psi_{1}(x) \leqq u(x) \leqq \psi_{2}(x),
$$

with $\psi_{1}(x)$ bounded from above, and $\psi_{2}(x)$ from below; as well as for the "soft obstacle" problem, namely when

$$
a \leqq \int_{0} \zeta(x) u(x) d x \leqq b
$$

for a given positive function $\zeta(x)$. The above includes the problem with fixed volume, when $\zeta=1$ and $a=b$.

Combinations of these and other conditions may also be imposed, as long as they are compatible with $|u| \leqq T$ for large $T$.

2. The structure of the sets $P$ and $N$. The generalized solution $u$ may well take the values $+\infty$ and $-\infty$. We set

$$
\begin{aligned}
& P=\{x \in \Omega: u(x)=+\infty\} \\
& N=\{x \in \Omega: u(x)=-\infty\} .
\end{aligned}
$$

The purpose of this section is to study the properties of the above sets.

Since we want to treat the obstacle problem, and even other situations such as the soft obstacles, we begin by observing that Proposition 1.2 remains valid, with the same proof, if $U_{j}$ are subsolutions, provided we add to the hypotheses the assumption that $U_{j}$ form a monotone decreasing sequence: $U_{j} \supset U_{j+1}$. In this case the limit $U$ will be a subsolution itself.

We shall concentrate on the set $P$; we remark however that if $u$ is a supersolution for $\mathscr{F}$ and if we set $H^{\prime}(x, t)=-H(x,-t)$ and $\gamma^{\prime}(x, t)=-\gamma(x,-t)$, the function $-u$ is a subsolution for $\mathscr{F}^{\prime}$, and hence every result concerning the set $P$ can be translated at once 
into a similar result concerning the set $N$ for supersolutions.

Proposition 2.1. Let $u$ be a subsolution for $\mathscr{F}$, and let

$$
H_{\infty}(x)=\lim _{t \rightarrow \infty} H(x, t): \quad \gamma_{\infty}(x)=\lim _{t \rightarrow \infty} \gamma(x, t) .
$$

Then $P$ is a subsolution for the functional

$$
G(P)=\int_{\Omega}\left|D \varphi_{P}\right|+\int_{\Omega} H_{\infty} \rho_{P} d x+\int_{i \Omega \Omega} \gamma_{\infty} \rho_{P} d H_{n-1} .
$$

Proof. For $j \in N$, let

$$
U_{j}=\{(x, t) \in Q: t<u(x)-j\} .
$$

The set $U_{j}$ is obviously a subsolution for

$$
F^{j}(A)=\int_{Q}\left|D \varphi_{A}\right|+\int_{Q} H_{j} \varphi_{A} d x d t+\int_{\partial Q} \gamma_{j} \varphi_{A} d H_{n}
$$

with

$$
H_{j}(x, t)=H(x, t+j) ; \quad \gamma_{j}(x, t)=\gamma(x, t+j) .
$$

We have $U_{j} \supset U_{j+1} \supset \cdots \supset W=\bigcap_{j \in N} U_{j}$, and hence by Proposition 1.2 and the remark above the set $W$ is a subsolution for the functional

$$
\int_{Q}\left|D \varphi_{W}\right|+\int_{Q} H_{\infty} \varphi_{W} d x d t+\int_{\partial Q} \gamma_{\infty} \varphi_{W} d H_{n}
$$

Since $W$ is a vertical cylinder, $W=P \times \boldsymbol{R}$, and since $H_{\infty}$ and $\gamma_{\infty}$ are independent of $t$, it follows easily that $P$ is a subsolution for $G$.

Before proceeding further in the discussion of the set $P$, we recall that if $E$ is a set and $x_{0} \in \partial E$, we say that $x_{0}$ belongs to the reduced boundary of $E$ if for every $R>0$ we have:

$$
0<\left|E \cap B_{R}\left(x_{0}\right)\right|<\left|B_{I_{i}}\left(x_{0}\right)\right|
$$

where $B_{R}\left(x_{0}\right)$ is the ball of radius $R$ centred at $x_{0}$. It is well known that after changing $E$ in a set of measure zero we may suppose that $\partial E$ coincides with the essential boundary of $E$.

THEOREM 1.1. Let $U$ be a local subsolution for the functional $F$, and suppose that the boundary of $U, \partial U$, coincides with the essential boundary. Then the same is true for $P$.

Proof. It is clearly sufficient to show that the boundary of $W=P \times \boldsymbol{R}$ is essential. Moreover we have only to show the first 
of inequalities (21), since the second follows at one from the remark that $W=\bigcap_{j \in N} U_{j}$.

Actually, we shall prove a stronger result, namely that if $z_{0}=\left(x_{0}, t_{0}\right)$ is a point in $Q$ and if for all $r>0$ we have $\left|U_{r}\left(z_{0}\right)\right|>0$, then for $r$ small enough $\left|U_{r}\left(z_{0}\right)\right| \geqq c_{3} r^{n+1}$, where we have set

$$
U_{r}\left(z_{0}\right)=U \cap C_{r}\left(z_{0}\right) ; \quad C_{r}\left(z_{0}\right)=\left\{z=(x, t):\left|z-z_{0}\right|<r,\left|t-t_{0}\right|<r\right\} .
$$

Let $z_{0} \in Q$ and let $0<r<R<\operatorname{dist}\left(z_{0}, \partial Q\right)$. Since $U$ is a subsolution we have, for large $T, F_{T}(U) \leqq F_{T}\left(U-C_{r}\right)$, and therefore:

$$
\int_{C_{r}}\left|D \varphi_{U}\right|+\int_{C_{r}} H \varphi_{U} d x d t \leqq \int_{\partial C_{r}} \varphi_{U} d H_{n} .
$$

On the other hand we have for almost every $r<R$ :

$$
\int\left|D \varphi_{U_{r}}\right|=\int_{C_{r}}\left|D \varphi_{U}\right|+\int_{\partial C_{r}} \varphi_{U} d H_{n}
$$

and whence

$$
\int\left|D \varphi_{U_{r}}\right|+\int H{\varphi_{U_{r}}} d x d t \leqq 2 \int_{\partial C_{r}} \varphi_{U} d H_{n}
$$

Suppose now that we have $t>0$ in $C_{r}$ (or in other words that $\left.t_{0}>R\right)$. Then

$$
\int H \varphi_{U_{r}} d x d t \geqq \int H_{0}^{-} \varphi_{U_{r}} d x d t
$$

where $H_{0}^{-}(x)=\min (H(x, 0), 0)$. From Lemma 2.1 below we get

$$
\int H \varphi_{U_{r}} d x d t \geqq-k(n)\left\|H_{0}^{-}\right\|_{n, B_{R}} \int\left|D \varphi_{U_{r}}\right|
$$

and hence

$$
\left(1-k(n)\left\|H_{0}^{-}\right\|_{n, B_{R_{n}}}\right) \int\left|D \varphi_{C^{\prime}}\right| \leqq 2 \int_{\partial C_{r}} \varphi_{V} d H_{n},
$$

where we have denoted by $k(n)$ the isoperimetric constant in $\boldsymbol{R}^{n}$. The right-hand side of (2.6) represents the derivative of $\left|U_{r}\right|$, so that we have:

$$
\begin{aligned}
\frac{d}{d r}\left|U_{r}\right| & =\int_{\partial C_{r}} \varphi_{U} d H_{n} \geqq \frac{1}{2}\left(1-k(n)|| H_{0}^{-} \|_{n, B_{R}}\right) \int\left|D \varphi_{U_{r}}\right| \\
& \geqq \frac{1}{2 k(n+1)}\left(1-k(n)|| H_{0}^{-} \|_{n, B_{R}}\right)\left|U_{r}\right|^{n / n+1} .
\end{aligned}
$$

Let now $R$ be so small that $\left\|H_{0}^{-}\right\|_{n_{, B_{R}}}<1 / 2 k(n)$; then for almost every $r<R$ we get 


$$
\frac{d}{d r}\left|U_{r}\right| \geqq \frac{1}{4 k(n+1)}\left|U_{r}\right|^{n / n+1}
$$

and in conclusion, if $\left|U_{r}\right|>0$ for every $r$, we obtain

$$
\left|U_{r}\right| \geqq r^{n+1} / 4(n+1) k(n+1) .
$$

The estimate (2.7) holds obviously for each of the sets $U_{j}$ defined in Proposition 2.1, with $R$ independent of $j$. We may then pass to the limit as $j \rightarrow \infty$, getting the same estimate for $W$.

Finally, if $x_{0} \in \Omega$ is such that $\left|P_{r}\left(x_{0}\right)\right|=\left|P \cap B_{r}\left(x_{0}\right)\right|>0$ for every $r>0$, we conclude that

$$
\left|P_{r}\left(x_{0}\right)\right| \geqq r^{n} / 8(n+1) k(n+1) .
$$

We note that the above estimate (2.8) does not hold for supersolutions, and the Theorem 2.1 is in general false. For example the function $|x|^{-1}$ is a supersolution for small $|x|(H=0)$, and $P=\{0\}$.

To conclude the proof of the theorem it remains to prove the estimate (2.5). This is done in the next lemma.

Lemma 2.1. Let $h(x) \in L^{n}(\Omega)$ and let $E \subset C_{R}=B_{R} \times I_{R}$. Then

$$
\int_{E}|h| d x d t \leqq k(n)\|h\|_{n, B_{R}} \int\left|D \phi_{E}\right| .
$$

Proof. We have from Hölder's inequality

$$
\int_{E}|h| d x d t \leqq\|h\|_{n, B_{R}} \int\left|E_{t}\right|^{1-1 / n} d t
$$

where

$$
E_{t}=\{x \in \Omega:(x, t) \in E\} .
$$

On the other hand from the isoperimetric inequality we get

$$
\left.E_{t}\right|^{1-1 / n} \leqq k(n) \int\left|D \varphi_{E_{t}}\right|
$$

and therefore

$$
\int_{E}|h| d x d t \leqq k(n)\|h\|_{n, B_{R}} \int d t \int\left|D \varphi_{E_{t}}\right| \leqq k(n)\|h\|_{n, B_{R}} \int\left|D \varphi_{E}\right|
$$

3. Variational solutions. In general the sets $P$ and $N$ are not empty, and sometimes they may cover the whole of $\Omega$. The purpose of this section is to investigate under what conditions we may conclude the absence of these singular sets. 
We begin with two simple remarks concerning subsolutions; similar results hold for supersolutions.

(A) If $A$ is an open set in $\Omega$, and if the measure of $P \cap A$ is zero, then $P \cap A$ is empty. Actually we may say more, namely if $A \Subset \Omega$ and if $|P \cap A|$ is small enough (depending on $A$ and $H$ ) then $P \cap A=\varnothing$.

(B) If $P \cap A=\varnothing$, then $u$ is locally bounded from above in $A$. Assertion (B) follows from estimate (2.7), whereas (A) is a consequence of (2.8).

EXAMPLE 3.1. (Emmer [3]) Let $u(x)$ be a generalized solution for $\mathscr{F}$ with obstacles, i.e., satisfying the conditions

$$
\psi_{\lambda}(x) \leqq u(x) \leqq \psi_{2}(x)
$$

Suppose that $\psi_{1}(x) \leqq M_{1}$, and $\psi_{2}(x) \geqq M_{2}$. It is clear that $u(x)$ is a generalized subsolution in $\Omega \times\left(M_{1},+\infty\right)$, and a generalized supersolution in $\Omega \times\left(-\infty, M_{2}\right)$.

It follows from remarks (A) and (B) above that if the obstacle $\psi_{1}\left[\psi_{2}\right]$ is finite almost everywhere in $\Omega$, then $u$ will be locally bounded from below [from above].

When no obstacle is present we need some assumptions on $H$ and $\gamma$. Let us begin with some necessary conditions.

Suppose $u(x)$ is a smooth solution for $\mathscr{F}$ in $\Omega$. If $A \subset \Omega$, and we compare $\mathscr{F}(u)$ with $\mathscr{F}\left(u+t \varphi_{A}\right), t>0$, we get easily

$$
\int_{\Omega} T u \cdot D \varphi_{A}+\int_{\Omega} H \varphi_{A} d x+\int_{\hat{o} \Omega} \gamma^{+} \varphi_{A} d H_{n-1} \geqq 0
$$

where $\gamma^{+}(x, t)=\lim _{s \rightarrow t}+\gamma(x, s)$.

Taking into account that $|T u|<1$ in $\Omega$, and arguing as in [7], $\S 1$, we get the inequality

$$
\int_{\Omega} H \varphi_{A} d x+\int_{\partial \Omega} \gamma^{+} \varphi_{A} d H_{n-1}>-\int_{\Omega}\left|D \varphi_{A}\right|
$$

and therefore

$$
\int_{\Omega} H_{\infty} \varphi_{A} d x+\int_{\partial \Omega} \gamma_{\infty} \varphi_{A} d H_{n-1}>-\int_{\Omega}\left|D \varphi_{A}\right|
$$

for every set $A \neq \varnothing, \Omega$. In a similar way:

$$
\int_{\Omega} H_{-\infty} \varphi_{A} d x+\int_{\partial \Omega} \gamma_{-\infty} \varphi_{A} d H_{n-1}<\int_{\Omega}\left|D \varphi_{A}\right|
$$

for $A \neq \varnothing, \Omega$.

THEOREM 3.1. Let the strict inequality (3.2) hold for every 
nonempty set $A$, and let $u$ be a generalized subsolution for $\mathscr{F}$. Then $P=\varnothing$.

Proof. By Proposition 2.1, $P$ is a subsolution for the functional

$$
G(P)=\int_{\Omega}\left|D \varphi_{P}\right|+\int_{\Omega} H_{\infty} \varphi_{P} d x+\int_{\partial \Omega} \gamma_{\infty} \varphi_{P} d H_{n-1} .
$$

On the other hand we have from (3.1), $G(A) \geqq 0$, the equality holding only for $A=\varnothing$. This implies immediately that $P$ is empty.

The same argument shows that if (3.3) holds, with strict inequality for $\Omega$, and if $u$ is a supersolution, then $N=\varnothing$.

Example 3.2. Discontinuous obstacles (De Acutis [2]).

Suppose that the obstacles $\psi_{1}$ and $\psi_{2}$ of Example 3.1 are finite only in some regular subsets $D_{1}$ and $D_{2}$ of $\Omega$. Arguing as before we may conclude that the generalized solution is locally bounded in the interior of these sets, from above in $D_{2}$ and from below in $D_{1}$. If in addition condition (3.2) is satisfied in $\Omega-D_{2}$, and (3.3) in $\Omega-D_{1}$, we may conclude that $P=N=\varnothing$ and therefore that $u$ is locally bounded in $\Omega$.

In general, even when $P=N=\varnothing$, the solution $u$ can go to $\pm \infty$ when $x$ approaches $\partial \Omega$ (see e.g., [8]).

However this possibility can be excluded if we make some additional assumptions on the boundary function $\gamma$. In the following we shall suppose that there exist constants $\theta_{0}>0$ and $\alpha, 0 \leqq \alpha<1$, such that

$$
\begin{aligned}
& \gamma(x, t) \geqq-\alpha \quad \forall x \in \partial \Omega, \quad \forall t>\theta_{0} \\
& \gamma(x, t) \leqq \alpha \quad \forall x \in \partial \Omega, \quad \forall t<-\theta_{0} .
\end{aligned}
$$

We note that (3.4), (3.5) correspond to a bounded boundary datum in the case of Dirichlet's boundary conditions $\left(\gamma(x, t)=1-2 \varphi_{F}(x, t)\right)$, whereas in the case of capillarity boundary conditions they are equivalent to $|\cos \theta| \leqq \alpha<1$.

With the help of (3.4) and (3.5) we can prove the following generalization of Theorem 2.1.

THEOREM 3.2. Let $U$ be a local subsolution for the functional $F$, and let $z_{0}=\left(x_{0}, t_{0}\right), t_{0}>\theta_{0}+1$, be a point of $\bar{Q}$ such that for every positive $r$ :

$$
\left|U_{r}\right|=\left|U \cap C_{r}\left(z_{0}\right)\right|>0 .
$$

Suppose further that (3.4) is satisfied. Then there exist constants 
$R_{0}>0$ and $c_{4}$ such that for every $r \leqq R_{0}$ we have:

$$
\left|U_{r}\right| \geqq c_{4}{ }^{n+1} \text {. }
$$

Proof. As in Theorem 2.1 we compare $U$ with $U-C_{r}$, getting

$$
\int_{Q \cap C_{r}}\left|D \varphi_{U}\right|+\int_{Q \cap C_{r}} H \varphi_{L} d x d t+\int_{\hat{\sigma} Q \cap C_{r}} \gamma \varphi_{U} d H_{n} \leqq \int_{\hat{o} C_{r}} \varphi_{U} d H_{n}
$$

and therefore for almost every $r$ :

$$
\int_{\Omega}\left|D \varphi_{U_{r}}\right|+\int_{Q} H \varphi_{U_{r}} d x d t+\int_{\partial Q} \gamma \varphi_{U} d H_{n} \leqq 2 \int_{\partial C_{r}} \rho_{U} d H_{n} .
$$

We have from (3.4):

$$
\int_{\partial Q} \gamma \varphi_{U_{r}} d H_{n} \geqq-\alpha \int_{\partial Q} \varphi_{U_{r}} d H_{n}
$$

for every $r<1$, and from Lemma 1.1:

$$
\begin{aligned}
\int_{\partial Q} \varphi_{U_{r}} d H_{n} \leqq \int_{Q}\left|D \varphi_{U_{r}}\right|+c_{1} \mid & U_{r}\left|\leqq \int_{Q}\right| D \varphi_{U_{r}} \mid \\
& +c_{1} l k(n+1)\left|U_{r}\right|^{1 / n+1} \int\left|D \varphi_{U_{r}}\right| .
\end{aligned}
$$

If $R<1$ is so small that $c_{1} k(n+1)\left|C_{R}\right|^{1 / n+1}<1 / 2$, we get for every $r<R$ :

$$
\int_{\partial \Omega} \mathcal{P}_{U_{r}} d H \leqq \frac{1+c_{1} k(n+1)\left|C_{R}\right|^{1 / n+1}}{1-c_{1} l k(n+1)\left|C_{R}\right|^{1 / n+1}} \int_{\Omega}\left|D \rho_{U_{r}}\right|
$$

and

$$
\int\left|D \varphi_{U_{r}}\right| \leqq \frac{2}{1-c_{1} k(n+1)\left|C_{R}\right|^{1 / n+1}} \int_{Q}\left|D \varphi_{U_{r}}\right|
$$

Finally, we may estimate the curvature term as in (2.5):

$$
\int_{Q} H \varphi_{U_{r}} d x d t \geqq-k(n)\left\|H_{0}^{-}\right\|_{n, B_{R}} \int\left|D \varphi_{\Gamma^{-},}\right|,
$$

and in conclusion

$$
\begin{aligned}
\int_{Q} H \varphi_{U_{r}} d x d t & +\int_{\hat{\partial} Q} \gamma \varphi_{U_{r}} d H_{n} \geqq-\left\{\alpha \frac{1+c_{1} k(n+1)\left|C_{R}\right|^{1 / n+1}}{1-c_{1} k(n+1)\left|C_{R}\right|^{1 / n+1}}\right. \\
& \left.+k(n)|| H_{0}^{-} \|_{n, B_{R}} \frac{2}{1-c_{1} k(n+1)\left|C_{R l}\right|^{1 / n+1}}\right\} \int_{Q}\left|D \varphi_{U_{r}}\right|
\end{aligned}
$$

Since $\alpha<1$, we may choose $R_{0}$ small enough that the right-hand 
side is bounded from below by $\frac{1}{2}-(1+\alpha) \int_{Q}\left|D \varphi_{U_{r}}\right|$. We remark that the constant $R_{0}$ depends only on $H$ through $\left\|H_{0}^{-}\right\|_{n, B_{R}}$ and therefore it is uniform for $H_{0}^{-}$in compact sets of $L^{n}(\Omega)$.

In conclusion we get

$$
\frac{d}{d \nu^{\circ}}\left|U_{r}\right|=\int_{\partial C_{r}} \varphi_{l^{\prime}} d H_{n} \geqq \frac{1}{2} \varepsilon_{0} \int_{Q}\left|D \varphi_{l^{\prime} r}\right| \geqq \frac{1}{8} \varepsilon_{0} \int\left|D \varphi_{U_{r}}\right| \quad\left(\varepsilon_{0}=(1-\alpha) / 2\right)
$$

and arguing as in Theorem 2.1 we conclude for $r<R_{0}$ :

$$
\left|U_{r}\right| \geqq c_{4} r^{n+1}, \quad c_{4}=\varepsilon_{0} / 8(n+1) .
$$

REMARK 3.1. It follows from (3.7) that for $r<R_{0}$ :

$$
\left|P_{r}\right|=\left|P \cap B_{r}\left(x_{0}\right)\right| \geqq \varepsilon_{0} r^{n} / 16(n+1)
$$

uniformly for $x_{0} \in \bar{\Omega}$.

In particular, there exists a constant $p_{0}$, depending only on $\alpha$ and on $H_{0}^{-}\left(p_{0}\right.$ is uniform for $H_{0}^{-}$in compact sets of $\left.L^{n}(\Omega)\right)$ such that $|P|<p_{0}$ implies that $P$ is empty and $u$ is bounded from above in the whole of $\Omega$. This makes possible to improve the results of Examples 3.1 and 3.2 .

EXAMPle 3.3. In Theorem 3.1 and in the above examples we have always made the assumption that the strict inequality (3.2) holds for $\Omega$ itself. It is easily seen that if the equality holds for $\Omega$, we cannot expect in general to have a bounded solution. For example, let $\Omega=\left\{x \in \boldsymbol{R}^{2}:|x|<1\right\}$, and let

$$
\begin{aligned}
H(x, u) & =\frac{2}{\pi} \operatorname{arctg} u-3 \\
\kappa(x, u) & =|u| .
\end{aligned}
$$

(Dirichlet problem with zero boundary data.) We have $H_{\infty}=-2$ and (3.2) is satisfied but the equality holds for $\Omega$. In this case we have $P=\Omega$, and $u=+\infty$.

REMARK 3.2. It is clear that a bounded solution is a variational solution, namely it has finite area and minimizes the functional $\mathscr{F}$ in $B V(\Omega)$. Moreover, if $H$ is Lipschitz-continuous the function $u(x)$ is of class $C^{2+\alpha}$ in $\Omega$ and is a classical solution of the equation

$$
\operatorname{div} T u=H(x, u) .
$$

REMARK 3.3. It follows from Theorem 3.2 that every generalized solution which is almost everywhere finite is bounded. In particular this is true for every $L^{1}$ generalized solution. It is easily 
seen that it is possible to give an estimate of $\sup u$ in terms of the $L^{1}$ norm of $u$, or better in terms of $\int_{\Omega} u^{+} d x, u^{+}=\max \left(u, \theta_{0}\right)$.

For, let $\theta_{0}$ and $R_{0}$ be as in Theorem 3.2, and let $x_{0} \in \Omega$ be such that

$$
\sup _{\Omega} u<u\left(x_{0}\right)+1 \text {. }
$$

For $j \in N$ let $z_{j}=\left(x_{0}, \theta_{0}+2 j R_{0}\right)$; we have $z_{j} \in U$ for $j \leqq k=$ $\left[u\left(x_{0}\right)-\theta_{0} / 2 R_{0}\right]$. From Theorem 3.2 we get

$$
\left|U_{R_{0}}\left(z_{j}\right)\right| \geqq c_{4} R_{0}^{n+1}
$$

and therefore

$$
\int_{\Omega} u^{+} d x \geqq \sum_{j=1}^{k}\left|U_{R_{0}}\left(z_{j}\right)\right| \geqq k c_{4} R_{0}^{n+1} .
$$

In conclusion

$$
\sup _{\Omega} u \leqq 1+u\left(x_{0}\right) \leqq \frac{2}{c_{4} R_{0}^{n}} \int_{\Omega} u^{+} d x+\theta_{0}+2 R_{0}+1 .
$$

We remark that estimate (3.11) holds for minima with obstacle $u \geqq \psi$, provided $\psi \leqq M \leqq \theta_{0}$. Moreover, the bound for sup $u$ depends only on $\int_{\Omega} u^{+} d x$ and on $R_{0}$ and $\theta_{0}$; it is therefore uniform for $H_{0}^{-}$in compact subset of $L^{n}(\Omega)$.

4. An application: Lagrange multipliers. We apply now the results of the previous sections to the discussion of the existence of Lagrange multipliers for minima with obstacle and constant volume.

Let $\psi(x)$ be a function bounded from above, and let $V$ be a real number, with

$$
V>\int_{\Omega} \psi d x \geqq-\infty .
$$

We have seen in $\S 1$ that the functional

$$
\mathscr{F}(u)=\int_{\Omega} \sqrt{1+|D u|^{2}}+\int_{\Omega} \lambda(x, u) d x+\int_{\partial \Omega} \kappa(x, u) d H_{n-1}
$$

with the constraints

$$
\begin{gathered}
u \geqq \psi \\
\int_{\Omega} u d x=V
\end{gathered}
$$

has a generalized solution; i.e., that there exists a function $u: \Omega \rightarrow[-\infty,+\infty]$ such that its subgraph $U$ minimizes locally in $Q$ 
the functional

$$
F(U)=\int_{Q}\left|D \varphi_{U}\right|+\int_{Q} H \varphi_{l} d x d t+\int_{\partial Q} \gamma \varphi_{U} d H_{r}
$$

among all subsets $E \subset Q$ such that $E \supset \Psi$ and $\left|E^{+}\right|-\left|E^{-}\right|=V$

$$
\left(E^{ \pm}=\{z=(x, t) \in Q: z \in E, t \gtrless 0\}\right) .
$$

Our goal is to show that there exists a variational solution to the above problem, and moreover that such solution may be obtained as a minimum for the functional

$$
\mathscr{F}_{q}(u)=\mathscr{F}(u)-q \int_{\Omega} u d x
$$

for a suitable value of the constant $q$ (the Lagrange multiplier).

Our hypotheses will be those of Theorem 3.2, namely:

(i) $\lambda(x, u)$ and $\kappa(x, u)$ are convex functions in $u$, or, what is the same, $H(x, u)$ and $\gamma(x, u)$ are nondecreasing functions of $u$.

(ii) $H(x, t)$ is in $L^{n}(\Omega)$ for every $t \in \boldsymbol{R}$.

(iii) There exist constants $\theta_{0}$ and $\alpha, 0 \leqq \alpha<1$, such that

$$
\begin{aligned}
& \gamma(x, t) \geqq-\alpha \quad \forall x \in \Omega, \quad \forall t>\theta_{0} \\
& \gamma(x, t) \leqq \alpha \quad \forall x \in \Omega, \quad \forall t<-\theta_{0} .
\end{aligned}
$$

Of course we may assume that $\theta_{0} \geqq M=\sup \psi$.

For what concerns the obstacle $\psi$ we shall assume that it is an upper bounded measurable function, almost everywhere finite. In this way every solution $u_{q}$ for the functional $\mathscr{F}_{q}$ is bounded from below (see Remark 3.1).

To prove our result we have to show that there exist a value $q_{0}$ and a variational solution $u_{0}$ to $\mathscr{F}_{q_{0}}$ such that $\int_{\Omega} u_{0} d x=V$.

Lemma 4.1. Let $h(x)$ be in $L^{n}(\Omega)$ and let $\gamma(x) \geqq-\alpha$. Then for every set $A \subset \Omega$, satisfying

$$
c_{1} k(n)|A|^{1 / n}<1
$$

we have

$$
\begin{gathered}
\int_{A} h d x+\int_{\partial \Omega \Omega} \gamma \varphi_{A} d H_{n-1} \geqq-\left\{\alpha \frac{1+c_{1} k(n)|A|^{1 / n}}{1-c_{1} k(n)|A|^{1 / n}}\right. \\
\left.+\frac{2 k(n)\|h\|_{n, A}}{1-c_{1} k(n)|A|^{1 / n}}\right\} \int_{\Omega}\left|D \varphi_{A}\right|
\end{gathered}
$$

Proof. We proceed as in Theorem 3.2. We have 


$$
\int_{\partial \Omega} \gamma \varphi_{A} d H_{n-1} \geqq-\alpha \int_{\partial \Omega} \varphi_{A} d H_{n-1} .
$$

From Lemma 1.1

$$
\int_{\partial \Omega} \varphi_{A} d H_{n-1} \leqq \int_{\Omega}\left|D \varphi_{A}\right|+c_{1}|A| \leqq \int_{\Omega}\left|D \varphi_{A}\right|+c_{1} k(n)|A|^{1 / n} \int\left|D \varphi_{A}\right|
$$

and therefore, if $c_{1} k(n)|A|^{1 / n}<1$,

$$
\begin{aligned}
& \int_{\partial \Omega} \varphi_{A} d H_{n-1} \leqq \frac{1+c_{1} k(n)|A|^{1 / n}}{1-c_{1} k(n)|A|^{1 / n}} \int_{\Omega}\left|D \varphi_{A}\right| \\
& \int\left|D \varphi_{A}\right| \leqq \frac{2}{1-c_{1} k(n)|A|^{1 / n}} \int_{\Omega}\left|D \varphi_{A}\right| .
\end{aligned}
$$

Moreover

$$
\int_{A} h d x \geqq-\|h\|_{n, A} \frac{2 k(n)}{1-c_{1} k(n)|A|^{1 / n}} \int_{\Omega}\left|D \varphi_{A}\right|
$$

from which (4.6) follows at once.

A consequence of Lemma 4.1 is that, at least for $q$ negative and big enough, the functional $\mathscr{F}_{q}$ has a variational solution. For that it is sufficient to show that condition (3.2) holds (with strict inequality even for $\Omega$ ) when $H_{\infty}$ is replaced by $H_{\infty}-q$. We distinguish two cases, desending on the size of $A$. If $A$ is small, we have using Lemma 4.1:

$$
\begin{aligned}
& \int_{A}\left(H_{\infty}-q\right) d x+\int_{\partial \Omega} \gamma_{\infty} \varphi_{A} d H_{n-1} \geqq \int_{A} H_{0}^{-} d x+\int_{\partial \Omega} \gamma_{\infty} \varphi_{A} d H_{n-1} \\
&<-\int_{\Omega}\left|D \varphi_{A}\right|
\end{aligned}
$$

provided $|A|$ is smaller than some constant $\sigma_{0}$ depending only on $\alpha$ and $H_{0}^{-}$.

On the other hand, if $|A|>\sigma_{0}$ we have:

$$
\begin{aligned}
\int_{A}\left(H_{\infty}-q\right) d x+ & \int_{\partial \Omega} \gamma_{\infty} \varphi_{A} d H_{n-1} \geqq-\alpha \int_{\Omega}\left|D \varphi_{A}\right|-c_{1}|A| \\
& +\int_{\Omega} H_{0}^{-} d x-q|A|>-\int_{\Omega}\left|D \varphi_{A}\right|
\end{aligned}
$$

whenever $-\left(q+\alpha c_{1}\right) \sigma_{0}+\int_{\Omega} H_{0}^{-} d x \geqq 0$.

Let us denote by $S$ the set of all the values of $q$ for which the necessary condition

$$
\int_{A}\left(H_{\infty}-q\right) d x+\int_{\partial \Omega} \gamma_{\infty} \varphi_{A} d H_{n-1} \geqq-\int_{\Omega}\left|D \varphi_{A}\right|
$$


is satisfied for all $A \subset \Omega$, and let $q_{0}=\sup S$.

It is clear from the above that $S \neq \varnothing$ and therefore $q_{0}>-\infty$. It is easily seen that (4.7) holds for $q_{0}$ itself, and that for $q<q_{0}$ we have the strict inequality for every nonempty set, including $\Omega$ itself. It follows that $\mathscr{F}_{q}$ has bounded (variational) solutions for all $q<q_{0}$ (and possibly for $q_{0}$ ), but not for any $q>q_{0}$, so that $S=\left(-\infty, q_{0}\right]$.

For $q<q_{0}$ we define $V(q)$ as the set described by the integral

$$
\int_{\Omega} u d x
$$

when $u$ varies among all solutions to $\mathscr{F}_{q}$. Since $\mathscr{F}_{q}$ is convex (but not strictly convex), the set $V(q)$ is a closed interval, which may of course reduce to a point.

Lemma 4.2. Let $p<q$ and let $u, v$ be solutions of $\mathscr{F}_{q}, \mathscr{F}_{q}$ respectively. Then $u \leqq v$.

Proof. It is well known (see [1] and [6], Theorem 1.17) that for every $w \in B V(\Omega)$ there exists a sequence $w_{j} \in C^{\infty} \cap B V(\Omega)$ such that $w_{j} \rightarrow w$ in $L^{1}(\Omega)$ and $\sqrt{1+\left|D w_{j}\right|^{2}} \rightarrow \sqrt{1+|D w|^{2}}$. Moreover $w_{j}=w$ on $\partial \Omega$, and if $|w| \leqq M$ we may choose the $w_{j}$ satisfying $\left|w_{j}\right| \leqq M$.

It is now a simple matter of computation to show that for any $r, \mathscr{F}_{r}\left(w_{j}\right) \rightarrow \mathscr{F}_{r}(w)$. In conclusion, for every $\varepsilon>0$ there exist $C^{\infty}$-functions $u_{\varepsilon}$ and $v_{\varepsilon}$ such that

$$
\begin{aligned}
& \left\|u_{\varepsilon}-u\right\|_{1}+\left\|v_{\varepsilon}-v\right\|_{1}<\varepsilon \\
& \mathscr{F}_{p}\left(u_{\varepsilon}\right)<\mathscr{F}_{p}(u)+\varepsilon \\
& \mathscr{F}_{q}\left(v_{\varepsilon}\right)<\mathscr{F}_{q}(v)+\varepsilon .
\end{aligned}
$$

Let $A=\left\{x \in \Omega: u_{\varepsilon}(x)>v_{\varepsilon}(x)\right\}$; we have

$$
\begin{gathered}
\mathscr{F}_{p}\left(\min \left(u_{\varepsilon}, v_{\varepsilon}\right)\right)=\mathscr{F}_{p}\left(u_{\varepsilon}\right)+\int_{A} \sqrt{1+\left|D v_{\varepsilon}\right|^{2}}-\int_{A} \sqrt{1+\left|D u_{\varepsilon}\right|^{2}} \\
+\int_{A}\left[\lambda\left(x, v_{\varepsilon}\right)-\lambda\left(x, u_{\varepsilon}\right)\right] d x+\int_{\partial 2 \cap \cap A}\left[\kappa\left(x, v_{\varepsilon}\right)-\kappa\left(x, u_{\varepsilon}\right)\right] d H_{n-1} \\
-p \int_{A}\left(v_{\varepsilon}-u_{\varepsilon}\right) d x \geqq \mathscr{F}_{p}(u) \geqq \mathscr{F}_{p}\left(u_{\varepsilon}\right)-\varepsilon .
\end{gathered}
$$

Similarly:

$$
\begin{array}{r}
\mathscr{F}_{q}\left(\min \left(u_{\varepsilon}, v_{\varepsilon}\right)\right)=\mathscr{F}_{q}\left(v_{\varepsilon}\right)-\int_{A} \sqrt{1+\left|D v_{\varepsilon}\right|^{2}}+\int_{A} \sqrt{1+\left|D u_{\varepsilon}\right|^{2}} \\
\quad-\int_{A}\left[\lambda\left(x, v_{\varepsilon}\right)-\lambda\left(x, u_{\varepsilon}\right)\right] d x-\int_{\partial \Omega \cap A}\left[\kappa\left(x, v_{\varepsilon}\right)-\kappa\left(x, u_{\varepsilon}\right)\right] d H_{x-1}
\end{array}
$$




$$
+q \int_{A}\left(v_{\varepsilon}-u_{\varepsilon}\right) d x \geqq \mathscr{F}_{q}(v) \geqq \mathscr{F}_{q}\left(v_{\varepsilon}\right)-\varepsilon .
$$

In conclusion, we have

$$
(q-p) \int_{A}\left(u_{s}-v_{\varepsilon}\right) d x=(q-p) \int_{\Omega}\left(\max \left(u_{\varepsilon}, v_{\varepsilon}\right)-v_{\varepsilon}\right) d x<2 \varepsilon
$$

and passing to the limit as $\varepsilon \rightarrow 0$ :

$$
(q-p) \int_{\Omega}(\max (u, v)-v) d x \leqq 0
$$

which implies $\max (u, v)=v$ and therefore $u \leqq v$.

A trivial consequence of the above lemma is that if $q_{1}<q_{2}$ and $u_{1}, u_{2}$ are solutions of $\mathscr{F}_{q_{1}}, \mathscr{F}_{q_{2}}$ respectively, we have

$$
\int_{\Omega} u_{1} d x \leqq \int_{\Omega} u_{2} d x
$$

In other words, the mapping $q \rightarrow V(q)$ is nondecreasing.

Lemma 4.3. For every $V$, $\inf _{q<q_{0}} V(q)<V<\sup _{q<q_{0}} V(q)$, there exists a $q^{\prime}<q_{0}$ such that $V \in V\left(q^{\prime}\right)$.

Proof. Let $S_{V}$ denote the set of all $q$ such that $V(q)<V$; and let $q^{\prime}=\sup S_{V}$. Let $q_{j} \nearrow q^{\prime}$ and for each $j$ let $u_{j}$ be a solution for $\mathscr{F}_{q_{j}}$. It is easily seen that the functions $u_{j}$ are uniformly bounded from below, and therefore from above because the integrals $\int_{\Omega} u_{j} d x$ are uniformly bounded. Since $\mathscr{F}_{q_{j}}\left(u_{j}\right) \leqq \mathscr{F}_{q_{j}}(0)=|\Omega|$, we conclude that $u_{j}$ is a bounded sequence in $B V$ and hence, passing possibly to a subsequence, it converges (strongly in $L^{1}$ and weakly in $L^{n / n-1}$ ) to some function $u^{\prime}$. From Theorem 1.2 we deduce that $u^{\prime}$ is a solution for $\mathscr{F}_{q^{\prime}}$, and $\int_{\Omega} u^{\prime} d x \leqq V$.

In a similar way, starting with a sequence $p_{j}$ decreasing to $q^{\prime}$, we arrive to a solution $u^{\prime \prime}$ for $\mathscr{F}_{q}$, with $\int_{\Omega} u^{\prime \prime} d x \geqq V$. From the convexity of $\mathscr{F}_{q^{\prime}}$ it follows that $V\left(q^{\prime}\right)$ is an interval, and hence $V \in V\left(q^{\prime}\right)$.

To conclude our proof we have only to show that every value $V>\int_{\Omega} \psi d x$ is reached.

LEMMA 4.4. Let $\eta(x)$ be a smooth function in $\Omega$, such that $\eta \geqq$ s. Let $q_{j} \rightarrow-\infty$ and let $u_{j}$ be a solution of $\mathscr{F}_{q_{j}}$. Then 


$$
\lim _{j \rightarrow \infty} \max \left(u_{j}, \eta\right)=\eta
$$

Proof. Let $q_{1}<q_{0}$ and let $q<q_{1}$. Let $u$ be a solution of $\mathscr{F}_{q}$ and let $u_{\varepsilon}$ be as in Lemma 4.2. We may compare $u_{\varepsilon}$ with $v_{\varepsilon}=$ $\min \left(u_{\varepsilon}, \eta\right)$, getting

$$
\begin{aligned}
& q \int_{\Omega}\left(v_{\varepsilon}\right.\left.-u_{\varepsilon}\right) d x \leqq \int_{A} \sqrt{1+|D \eta|^{2}}-\int_{A} \sqrt{1+\left|D u_{\varepsilon}\right|^{2}} \\
& \quad+\int_{\Omega}\left[\lambda\left(x, v_{\varepsilon}\right)-\lambda\left(x, u_{\varepsilon}\right)\right] d x+\int_{\partial \Omega}\left[\kappa\left(x, v_{\varepsilon}\right)-\kappa\left(x, u_{\varepsilon}\right)\right] d H_{n-1}
\end{aligned}
$$

We have

$$
\int_{\Omega}\left[\lambda\left(x, v_{\varepsilon}\right)-\lambda\left(x, u_{\varepsilon}\right)\right] d x=-\int_{A} d x \int_{\eta}^{u_{\varepsilon}} H(x, t) d t \leqq-\int_{\Omega} H(x, \eta)\left(u_{\varepsilon}-v_{\varepsilon}\right) d x
$$

and

$$
\begin{array}{r}
\int_{\partial \Omega}\left[\kappa\left(x, v_{\varepsilon}\right)-\kappa\left(x, u_{\varepsilon}\right)\right] d H_{n-1} \leqq \alpha \int_{\partial \Omega}\left|v_{\varepsilon}-u_{\varepsilon}\right| d H_{n-1} \leqq \int\left|D\left(v_{\varepsilon}-u_{\varepsilon}\right)\right| \\
+c_{1} \int\left|v_{\varepsilon}-u_{\varepsilon}\right| d x \leqq \int_{A}|D \eta|+\int_{A}\left|D u_{\varepsilon}\right|+c_{1} \int\left|u_{\varepsilon}-v_{\varepsilon}\right| d x
\end{array}
$$

In conclusion:

$$
\begin{gathered}
-\left(c_{1}+q\right) \int_{\Omega}\left|u_{\varepsilon}-v_{\varepsilon}\right| d x=-\left(c_{1}+q\right) \int_{\Omega}\left(\max \left(u_{\varepsilon}, \eta\right)-\eta\right) d x \\
\leqq 2 \int_{A} \sqrt{1+|D \eta|^{2}}-\int_{\Omega} H(x, \eta)\left(u_{\varepsilon}-v_{\varepsilon}\right) d x
\end{gathered}
$$

and letting $\varepsilon \rightarrow 0$ :

$$
\begin{gathered}
-\left(c_{1}+q\right) \int_{\Omega}(\max (u, \eta)-\eta) d x \leqq 2 \int_{\Omega} \sqrt{1+|D \eta|^{2}} \\
+\int_{\Omega} H(x, \eta)(u-v) d x .
\end{gathered}
$$

On the other hand, it follows from Lemma 4.2 that $u=u_{q} \leqq u_{q_{1}}$, and therefore the right-hand side of (4.8) is bounded independently of $q$. Letting $q \rightarrow-\infty$ we get the conclusion of the theorem.

The above result shows that inf $V(q)=\int_{\Omega} \psi d x$. The existence of the Lagrange multiplier will follow if we show that $\sup V(q)=$ $+\infty$.

This is easy if $q_{0}=+\infty$, i.e., if the functional $\mathscr{F}_{q}$ has variational solutions for every real $q$ (this happens for instance if $H_{\infty}=+\infty$ ). In this case we have only to repeat the above argument to show that the volume $V(q)$ tends to infinity as $q \rightarrow+\infty$. More complicate is the case when $q_{0}<+\infty$. Let $q_{j}$ be an increasing sequence, $q_{j} \rightarrow q_{0}$, and let $u_{j}$ be the corresponding sequence of solutions. We distin- 
guish two cases.

$$
\left\|u_{j}\right\|_{1} \longrightarrow+\infty
$$

In this case, since $u_{j}$ is increasing, we have $V_{j}=\int_{\Omega} u_{j} d x \rightarrow+\infty$, and therefore $\sup V(q)=+\infty$.

$$
\left\|u_{j}\right\|_{1} \leqq M
$$

By Remark 3.3 the functions $u_{j}$ are uniformly bounded:

$$
\left|u_{j}(x)\right| \leqq M_{1}
$$

and therefore it is possible to extract from $u_{j}$ a subsequence which converges to a function $u(x)$, a variational solution for $\mathscr{F}_{q_{0}}$. It is then satisfied the necessary condition:

$$
-q_{0}|A|+\int_{A} H(x, u(x)) d x+\int_{\partial \Omega} \gamma^{+}(x, u(x)) \varphi_{A} d H_{n-1} \geqq-\int_{\Omega}\left|D \varphi_{A}\right|
$$

for every set $A \subset \Omega$.

On the other hand from the true definition of $q_{0}$ we have:

$$
-q_{0}|A|+\int_{A} H_{\infty} d x+\int_{\hat{\partial} \Omega} \gamma_{\infty} \varphi_{A} d H_{n-1} \geqq-\int_{\Omega}\left|D \varphi_{A}\right|
$$

LEMMA 4.5. Let the strict inequality hold in (4.10) for every nonempty set $A$. Then there exists $q>q_{0}$ such that

$$
-q|A|+\int_{A} H_{\infty} d x+\int_{\partial \Omega} \gamma_{\infty} \varphi_{A} d H_{n-1} \geqq-\int_{\Omega}\left|D \varphi_{A}\right|
$$

for every $A \subset \Omega$.

Proof. Let $q_{j}=q_{0}+1 / j$, and suppose that for every $j$ there exists a set $A_{j}$ such that

$$
\text { (4.11) }-q_{j}\left|A_{j}\right|+\int_{A_{j}} H_{\infty} d x+\int_{\partial \Omega} \gamma_{\infty} \Phi_{A_{j}} d H_{n-1}<-\int_{\Omega}\left|D \varphi_{A_{j}}\right|
$$

From (4.11) we get

$$
\int_{\Omega}\left|D \varphi_{A_{j}}\right| \leqq\left|q_{j}\right||\Omega|+\int_{\Omega} H_{\infty}^{-} d x+H_{n-1}(\partial \Omega)
$$

and therefore, passing to a subsequence we may conclude that $A_{j} \rightarrow A$. From (4.6) with $h=H_{\infty}^{-}-q_{j}$, we conclude that the measure of $A_{j}$ is bounded away from zero, and whence $A$ has positive measure. Passing to the limit as $j \rightarrow+\infty$ we get:

$$
-q_{0}|A|+\int_{A} H_{\infty} d x+\int_{\partial \Omega} \gamma_{\infty} \varphi_{A} d H_{n-1} \leqq-\int_{\Omega}\left|D \varphi_{A}\right|
$$


contradicting the assumption of the lemma.

It follows from the above lemma and the definition of $q_{0}$ that the equality sign must hold in (4.10) for some nonempty set $A$. From the monotonicity of $H$ and $\gamma$ and from (4.9) we get then:

$$
-q_{0}|A|+\int_{A} H(x, u(x)) d x+\int_{\partial \Omega} \gamma^{+}(x, u(x)) \varphi_{A} d H_{n-1}=-\int_{\Omega}\left|D \varphi_{A}\right|
$$

and therefore for almost every $x \in A$ and for every $t>u(x)$ :

$$
\begin{aligned}
& H(x, t)=H_{\infty}(x) \\
& \gamma(x, t)=\gamma_{\infty}(x) .
\end{aligned}
$$

In particular we may conclude that $\mathscr{F}_{q_{0}}\left(u+c \varphi_{A}\right)=\mathscr{F}_{q_{0}}(u)$ for every positive constant $c$, and hence

$$
V\left(q_{0}\right)=\left[\int_{\Omega} u d x,+\infty\right) .
$$

In any case we have then

$$
V(S)=\left(\int_{\Omega} \psi d x,+\infty\right]
$$

thus proving the existence of a Lagrange multiplier.

\section{REFERENCES}

1. G. Anzellotti and M. Giaquinta, Funzioni $B V$ e tracce, to appear in Rend. Sem. Mat. Padova.

2. D. De Acutis, Superfici non parametriche a curvatura media assegnata con ostacoli, Ann. Univ. Ferrara, 23 (1977), 225-250.

3. M. Emmer, Superfici di curvatura media assegnata con ostacolo, Ann. Mat. Pura ed Appl. S. IV, 109 (1976), 371-389.

4. C. Gerhardt, On the capillarity problem with constant volume, Ann. Sc. Norm. Sup. Pisa S. IV, 2 (1975), 303-320.

5. E. Giusti, Boundary value problems for non-parametric surfaces of prescribed mean curvature, Ann. Sc. Norm. Sup. Pisa S. IV, 3 (1976), 501-548.

6. Minimal surfaces and function of bounded variation, Notes on pure mathematics, 10. Canberra, 1977.

7. — On the equation of surfaces of prescribed mean curvature. Existence and uniqueness without boundary conditions, Inv. Math., 46 (1978), 111-137.

8. U. Massari, Problema di Dirichlet con dati infiniti per le superfici a curvature media assegnate, to appear.

9. M. Miranda, Superfici cartesiane generalizzate ed insiemi di perimetro finito sui prodotti cartesiani, Ann. Sc. Norm. Sup. Pisa S. III, 18 (1964), 515-542.

10. - Superfici minime illimitate, Ann. Sc. Norm. Sup. Pisa. S. IV, 4 (1977), 313-322.

11. Sulle singolarità eliminabili delle soluzioni dell'equazione delle superfici minime, Ann. Sc. Norm. Sup. Pisa S. IV, 4 (1977), 129-132. 
12. G. H. Williams, Surfaces of prescribed mean curvature with a volume constraint, to appear.

Received June 29, 1979. Partially supported by GNAFA-CNR.

Universita Di Pisa

56100 Pisa, Italy 



\section{PACIFIC JOURNAL OF MATHEMATICS}

\section{EDITORS}

DONALD BABBITT (Managing Editor)

University of California

Los Angeles, CA 90024

Hugo Rossi

University of Utah

Salt Lake City, UT 84112

C. C. MOORE and ANDREW OGG

University of California

Berkeley, CA 94720
J. DUGUNDJI

Department of Mathematics

University of Southern California

Los Angeles, CA 90007

R. FinN and J. Milgram

Stanford University

Stanford, CA 94305

\section{ASSOCIATE EDITORS}
E. F. BECKENBACH
B. H. NeumanN
F. WOLF
K. YoSHIDA

\section{SUPPORTING INSTITUTIONS}

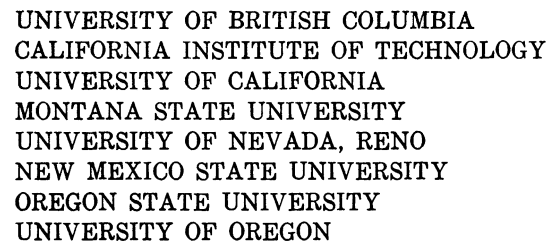

UNIVERSITY OF BRITISH COLUMBIA CALIFORNIA INSTITUTE OF TECHNOLOGY UNIVERSITY OF CALIFORNIA MONTANA STATE UNIVERSITY UNIVERSITY OF NEVADA, RENO NEW MEXICO STATE UNIVERSITY OREGON STATE UNIVERSITY UNIVERSITY OF OREGON

\author{
UNIVERSITY OF SOUTHERN CALIFORNIA \\ STANFORD UNIVERSITY \\ UNIVERSITY OF HAWAII \\ UNIVERSITY OF TOKYO \\ UNIVERSITY OF UTAH \\ WASHINGTON STATE UNIVERSITY \\ UNIVERSITY OF WASHINGTON
}

The Supporting Institutions listed above contribute to the cost of publication of this Journal, but they are not owners or publishers and have no responsibility for its content or policies.

Mathematical papers intended for publication in the Pacific Journal of Mathematics should be in typed form or offset-reproduced, (not dittoed), double spaced with large margins. Please do not use built up fractions in the text of the manuscript. However, you may use them in the displayed equations. Underline Greek letters in red, German in green, and script in blue. The first paragraph or two must be capable of being used separately as a synopsis of the entire paper. Please propose a heading for the odd numbered pages of less than 35 characters. Manuscripts, in triplicate, may be sent to any one of the editors. Please classify according to the scheme of Math. Reviews, Index to Vol. 39. Supply name and address of author to whom proofs should be sent. All other communications should be addressed to the managing editor, or Elaine Barth, University of California, Los Angeles, California, 90024.

50 reprints to each author are provided free for each article, only if page charges have been substantially paid. Additional copies may be obtained at cost in multiples of 50 .

The Pacific Journal of Mathematics is issued monthly as of January 1966. Regular subscription rate: $\$ 84.00$ a year (6 Vols., 12 issues). Special rato: $\$ 42.00$ a year to individual members of supporting institutions.

Subscriptions, orders for numbers issued in the last three calendar years, and changes of address shoud be sent to Pacific Journal of Mathematics, P.O. Box 969, Carmel Valley, CA 93924, U.S.A Old back numbers obtainable from Kraus Periodicals Co., Route 100, Millwood, NY 10546.

PUBLISHED BY PACIFIC JOURNAL OF MATHEMATICS, A NON-PROFIT CORPORATION

Printed at Kokusai Bunken Insatsusha (International Academic Printing Co., Ltd.). 8-8, 3-chome, Takadanobaba, Shinjuku-ku, Tokyo 160, Japan. 


\section{Pacific Journal of Mathematics \\ Vol. 88, No. $2 \quad$ April, 1980}

Reinhold Böhme, Stefan Hildebrandt and Engelbert Tausch, The two-dimensional analogue of the catenary ................. 247

Jean Ellen Taylor, Nonexistence of F-minimizing embedded disks ........ 279

Claus Gerhardt, A free boundary value problem for capillary surfaces ..... 285

Enrico Giusti, Generalized solutions for the mean curvature equation . . . . . 297

Jin-Tzu Chen, On the existence of capillary free surfaces in the absence of gravity.................................... 323

Leon M. Simon, Regularity of capillary surfaces over domains with corners.......................................... 363

Nicholas Jacob Korevaar, On the behavior of a capillary surface at a re-entrant corner.................................... 379

Henry Wente, The symmetry of sessile and pendent drops ............ 387

E. Gonzalez, Umberto Massari and I. Tamanini, Existence and regularity for the problem of a pendent liquid drop ...................... 399

Henry Wente, The stability of the axially symmetric pendent drop ........ 421

David Siegel, Height estimates for capillary surfaces . . . . . . . . . . . . 471

Bruce Edward Turkington, Height estimates for exterior problems of capillarity type ................................ 517

Robert Finn, The sessile liquid drop. I. Symmetric case .............. 541 